\title{
OPTICAL PROPERTIES OF HIGH REDSHIFT RADIO GALAXIES
}

\author{
Patrick J. McCarthy, Hyron Spinrad, and Wil van Breugel \\ Department of Astronomy, University of California \\ Berkeley, CA 94720 U.S.A.
}

The 3CR catalogue of extragalactic radio sources is now completely identified for $b>15^{\circ}$ and redshifts have been determined for $>98 \%$ of them (see Djorgovski et al. 1988 for the latest update). The radio galaxies in this catalogue span the redshift range from 0 to 2.48. This sample provides us with a unique opportunity to examine the optical and radio properties of a complete sample over a look-back time comparable to the Hubble time.

We begin by discussing the emission line properties. Interference filter images in the light of strong emission lines now exist for nearly 100 of the 210 3CR radio galaxies (Baum 1988; McCarthy 1989.). Based on these one can deduce a number of properties of the extended emission line regions (EELRs). At low redshifts $(\mathrm{z} \sim 0.2)$ the emission line gas tends to lie at distances of typically $10 \mathrm{kpc}$ from the nucleus and shows a tendency to lie in the quadrants of the sky defined by the radio source axis (Baum and Heckman 1988). The EELRs do not often show a direct relation to the radio source, but in a number of cases the gas lies near the boundary of the radio lobes (Baum et al. 1988). At larger redshifts the EELRs are found on scales of up to $250 \mathrm{kpc}\left(\mathrm{H}_{0}=50, \mathrm{q}_{0}=0\right)$ and tend to lie within $15^{\circ}$ of the radio source axis, and in a substantial number of cases the emission line gas is directly associated with one of the two radio hot spots (McCarthy et al. 1987). At redshifts larger than 1 the gas is seen within $15^{\circ}$ of the radio axis in nearly all cases. In a number of cases we find emission line gas along the radio source axis but more than $10 \mathrm{kpc}$ beyond the radio hotspots.

The luminosities of the EELRs range from typically $\mathrm{L}([\mathrm{OII}] \lambda 3727) \sim 3 \times 10^{41} \mathrm{erg}$ $\mathrm{s}^{-1}$ at $\mathrm{z} \sim 0$, to $\sim 10^{44}$ at $\mathrm{z}>1$. The total emission line luminosities correlate with radio luminosity at low redshifts as shown by Baum and Heckman (1988). Considering the entire $3 \mathrm{CR}$ sample, we find that the [OII] $\lambda 3727$ luminosity correlates with the radio luminosity over nearly 5 decades in radio luminosity.

The excitation of the emission line gas, both nuclear and extended can be investigated with the aid of line ratio diagrams of the sort employed by Baldwin, Phillips, and Terlevich (1981). For this study we must have a spectral diagnostic that can be used over a large range in redshift. For this reason we use the [OII] $\lambda 3727$, [NeIII] $\lambda 3869$, and $[\mathrm{NeV}] \lambda 3426$ emission lines (McCarthy et al. 1988). The 3CR radio galaxies show a well defined locus in the [OII]-[NeIII]-[NeV] plane that follows the trend of power law photoionization models with the ionization parameter in the range $\log U \sim-2 \sim-3$. Terlevich and Melnick (1985), however, show that photoionization from a hot black body can produce similar results. The strong correlation between the radio source axis and the location of the emission line gas for the large redshift (and hence high power galaxies) argues for powerlaw photoionization from the nucleus over hot stars in many cases. The existence of large amounts of line emission beyond the radio hotspots suggests that direct interaction between the radio emitting plasma and the thermal gas is not the dominant physical process, but rather that it is the direction of the outflow from the nucleus that is preferred. This leads us 
to propose that there is an anisotropic ionizing source associated with the nucleus in some cases (van Breugel and McCarthy 1987). This type of phenomenon is well documented for nearby lower luminosity AGNs (Unger et al. 1987). At the largest redshifts we can actually observe the continuum down to rest wavelengths of $\sim 1000 \AA$. The extrapolation to ionizing radiation is then quite small. For $3 \mathrm{C} 256(\mathrm{z}=1.8)$, for example, we observe sufficient ionizing continuum from the hot stars to account for the observed Ly $\alpha$ emission (Spinrad 1988). Thus it appears that the nucleus plays a large role in photoionizing the gas at small and intermediate redshifts, but as one goes to large look-back times when star formation rates were quite large, UV from the hot stars themselves may play a large role.

Of all the observable properties of these objects, the stellar continuum undergoes the most dramatic changes with redshift. Some of this may be due to the changing band pass of our ground based window, but much of it is likely to be due to genuine evolution of the star formation rates and processes. While nearby radio galaxies are all giant elliptical galaxies with fairly normal morphologies, at large redshifts the galaxies take on rather unusually elongated and multi-modal morphologies (Spinrad and Djorgovski 1984; Lilly and Longair 1984; Le Fèvre et al. 1988). McCarthy et al. (1987) and Chambers et al. (1987) show that these elongated galaxies are aligned with their radio source axes. Photometric studies in the visual and near IR indicate that these objects are undergoing large bursts of star formation. These facts lead McCarthy et al. (1987) to suggest that the alignments are the result of excess star formation induced by outflow from the nucleus. Lilly (1989) has recently shown that the level of star formation activity is well correlated with the radio spectral index, and that those galaxies which show the alignment effect to the largest extent have the most star formation activity.

Thus it appears that as we go to larger and large look-back times the influence of the nucleus on its environment increases, both in stimulating star formation and in ionizing the gas directly. While locally there is evidence that the AGN phenomenon is related to environment in the sense that mergers and interactions can stimulate the nucleus, at large redshifts we see that the nucleus has an enormous impact on its environment on scales up to a few hundred kiloparsecs.

Baldwin, J., Phillips, M. M., and Terlevich, R. 1981, P. A. S. P., 93, 5.

Baum, S. 1988 Ph. D. Thesis, The University of Maryland.

Baum, S. et al. 1988, Ap. J. Suppl., in press.

Baum, S., and Heckman, T. 1988, Ap. J., in press.

Chambers, K. C., Miley, G. K., and van Breugel, W. J. M. 1987, Nature, 329, 604.

Djorgovski et al., 1988, A. J., in press.

Le Fèvre, O., Hammer, F, Nottale, L., Mazure, A., and Christian C. 1988, Ap. J. Lett., 324, L1.

Lilly, S. J., and Longair, M. 1984, M. N. R. A. S., 211, 833.

Lilly, S. J. 1988, Ap. J., in press.

McCarthy, P. J. 1989 Ph. D. Thesis, The University of California.

McCarthy, P. J., van Breugel , W. J. M., Spinrad, H., and Djorgovski, S. 1987, Ap. J. Lett.,321, L29.

McCarthy, P. J., et al., 1988, in Cooling Flows in Clusters and Galaxies, A. C. Fabian ed., Kluwer Academic Publshers, in press.

Spinrad, H. and Djorgovski, S. 1984, P. A. S. P., 96, 795.

Spinrad, H. 1988 in Epoch of Galaxy Formation, C. F. Frenk ed., Reidel, in press.

Terlevich, R., and Melnick, J. 1985, M. N. R. A. S., 213, 831.

Unger, S. W. et al. 1987, M. N. R. A. S., 228, 671.

van Breugel, W. J. M. and McCarthy, P. J. 1987, in Proc. Conf. on Active Galactic Nucle, H. R. Miller and P. J. Witta eds., Spinger-Verlag. 


\section{DISCUSSION}

TSVETANOV Can you give some estimate of the viewing angle of the cones in which the ionizing UV radiation is emitted?

MCCARTHY At large redshift, $z \geq 0.8$, virtually all of the emission line gas lies within $15^{\circ}$ of the radio source axis, so I would estimate that the opening angle is of this order.

ANTONUCCI Might the extended optical continuum emission observed at $2 \mu$ be due to red supergiants rather than red giants?

MCCARTHY At present, all of the multicolor photometry is based on aperture measurements. The best fitting models, by Bruzual and Rocca-Volmerange, suggest that the $2 \mu$ flux is dominated by red giants. As yet, little $2 \mu$ photometry has been done on the extended continuum. 